\title{
Fracture through cavitation in a metallic glass
}

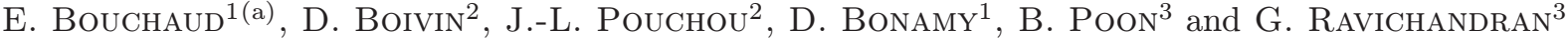 \\ ${ }^{1}$ C.E.A.-Saclay, DSM/DRECAM/SPCSI - 91191 Gif-Sur-Yvette Cedex, France, EU \\ ${ }^{2}$ ONERA, DMMP - 29 Avenue de la Division Leclerc, 92322 Châtillon Cedex, France, EU \\ ${ }^{3}$ California Institute of Technology, Division of Engineering and Applied Science - Pasadena, CA 91125, USA
}

received 3 February 2008; accepted in final form 4 August 2008

published online 11 September 2008

PACS 62.20.mm - Fracture

PACS 62.20.F--Deformation and plasticity

\begin{abstract}
The fracture surfaces of a Zr-based bulk metallic glass exhibit exotic multi-affine isotropic scaling properties. The study of the mismatch between the two facing fracture surfaces as a function of their distance shows that fracture occurs mostly through the growth and coalescence of damage cavities. The fractal nature of these damage cavities is shown to control the roughness of the fracture surfaces.
\end{abstract}

Copyright (c) EPLA, 2008

Introduction. - Since the pioneering work of Mandelbrot et al. [1], the statistical characterization of fracture surfaces has been a very active field of research. The fracture surfaces of various materials have shown surprising scaling properties (see for example [2-4] for reviews) over a wide range of length scales. Fracture profiles of materials as different as metallic alloys [5-10], silicate glasses [11,12], quasi-crystals [13], rocks [14], mortar [15], sea ice $[16]$, and wood $[17,18]$ have shown to be self-affine, with a roughness exponent $\zeta \approx 3 / 4$ in spite of huge differences in the fracture mechanisms. It was therefore suggested $[5,19]$ that $\zeta$ might have a universal value, i.e., independent of the fracture mode and of the material.

More recently, it has been shown $[11,13]$ that fracture surfaces are anisotropic, i.e. when profiles along the direction of crack propagation are considered, the roughness exponent is equal to $\beta \simeq 0.6$. Bonamy et al. [12] have shown that the set of exponents $\{\zeta \simeq 0.75, \beta \simeq 0.6\}$ define a universality class corresponding to length scales smaller than the process zone size, where non linear elastic processes take place. Above this process zone size, another university class is observed $[12,20,21]$ characterized by a set of exponents $\{\zeta \simeq 0.4, \beta \simeq 0.5\}$ that can be understood theoretically within the Linear Elastic Fracture Mechanics framework.

A third regime arises at very small length scales, characterized by a roughness index close to $\zeta \approx 0.5$, observed in a metallic alloy and in a soda-lime silicate glass [8-10] along a direction perpendicular to the direction of crack propagation. This regime was suggested [22] to be

\footnotetext{
(a) E-mail: elisabeth.bouchaud@cea.fr
}

characteristic of small damage cavities, i.e. of cavities small enough that they grow through surface enlargement rather than through front propagation.

In the present work, the morphology of the fracture surfaces of a bulk Zr-based metallic glass is investigated. We show that although these fracture surfaces are characterized by a roughness index close to 0.55 at all scales of observation, both in the directions perpendicular and parallel to crack propagation, they are clearly multi-affine. Through the mismatch between two opposite fracture surfaces, we analyze the appearance and growth of damage cavities. It is shown that their coalescence gives rise to fracture. Their fractal nature - their fractal dimension is close to 1.43 - is shown to be what triggers the roughness of the fracture surfaces.

Experiment. - The studied fracture surface is obtained from a Zr-based bulk metallic glass (Vitreloy 1) [23] notched sample subjected to uniaxial tension. Vitreloy 1 is one of these new bulk glass-forming metallic alloys with composition $\mathrm{Zr}_{41.2} \mathrm{Ti}_{13.8} \mathrm{Cu}_{12.5} \mathrm{Ni}_{10} \mathrm{Be}_{22.5}$, which exhibits high resistance with respect to crystallization in its wide supercooled liquid region. Its mechanical properties [24] — high strength to density ratio and high yield stress - make it a very good candidate for structural applications.

We use a dog-bone shape specimen $21.4 \mathrm{~mm}$ long in the $y$ tensile direction; Its basis has a surface of $3 \times 4.8 \mathrm{~mm}^{2}$ within the $x z$ fracture plane. The initial $0.5 \mathrm{~mm}$ notch lies along the $x$-axis and its front is parallel to the $z$-axis. Displacement of the grips is controlled at a strain rate of 


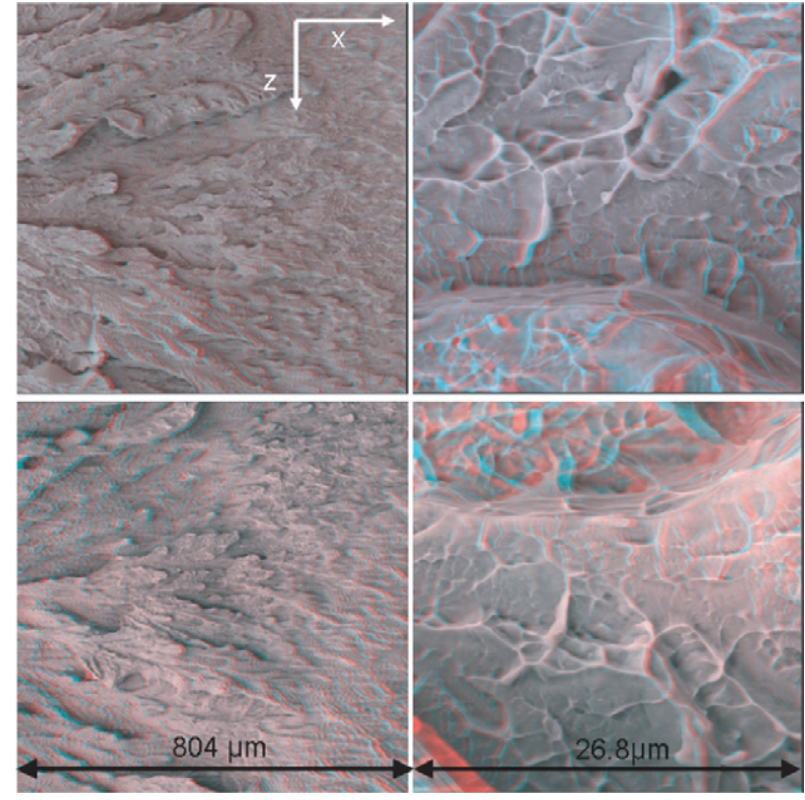

Fig. 1: Opposite fracture surfaces observed through stereo pairs with SEM. Left: the two opposite fracture surfaces (up and down) observed at magnification $\times 100$ (image size $804 \mu \mathrm{m}$ ); Right: magnification $\times 3000$ (image size $26.8 \mu \mathrm{m}$ ). $x$ is the direction of crack propagation, while the initial notch is parallel to the $z$-direction. $x z$ thus define the fracture plane.

$10^{-4} \mathrm{~s}^{-1}$. Fracture occurs for a stress of $1.9 \mathrm{GPa}$ and a strain of $2 \%$.

The morphology of the fracture surfaces is studied from digital elevation maps (DEM) obtained by 3D reconstruction from stereoscopic SEM images [25]. Two images of the same surface are recorded for two different tilt angles of the specimen, and treated by a specific software [25] to compute the elevation map of the scanned surface. In this experiment at all magnifications, Scanning Electron Microscope (SEM) pictures have been acquired at $0^{\circ}$ and $10^{\circ}$ (tilt angles), in 256 grey levels and $1024 \times 1024$ pixels size, on a Field Emission Gun SEM (FEG-SEM, ZEISS Gemini).

Each surface is studied at three magnifications for which DEMs are produced: $\times 100, \times 300$ and $\times 3000$. Figure 1 shows the two fracture surfaces at magnifications $\times 100$ and $\times 3000$. At low enough magnification $(\times 100$ - see fig. $1-$ and $\times 300$ ) the surfaces are obviously anisotropic in shape, with elongated leaf-like structures aligned along the direction $x$ of crack propagation.

Roughness analysis of the fracture surfaces. The roughness of the surfaces is estimated first along directions parallel and perpendicular to crack propagation, through the computation of the second-order moments $\Delta h(\Delta x)$ and $\Delta h(\Delta z)$ of height differences between points distant of $\Delta x$ (respectively, $\Delta z$ ) along (respectively, perpendicularly to) the direction $x$ of crack propagation. The results obtained for the six surfaces are gathered in the log-log plot of fig. 2. $\Delta h(\Delta x)$ (respectively,

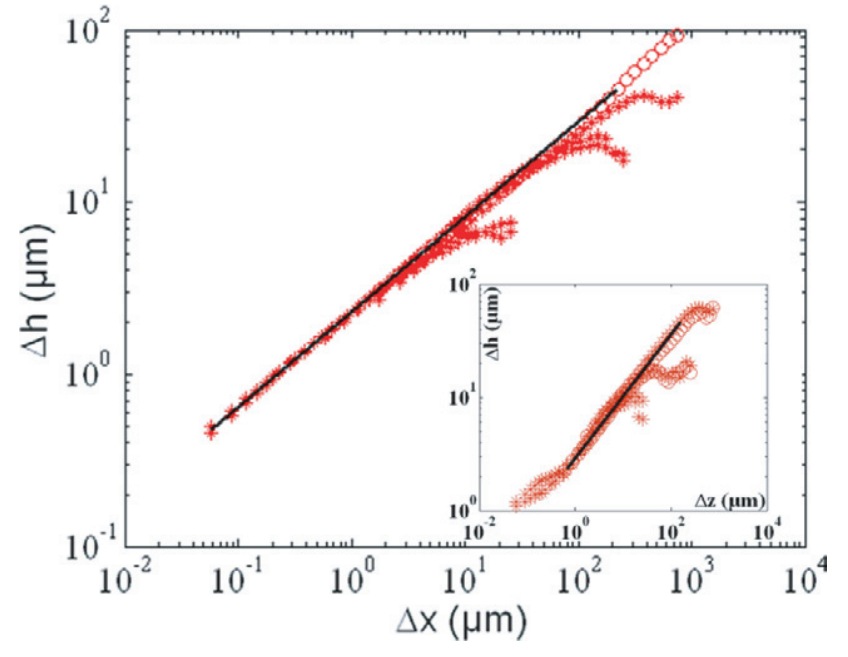

Fig. 2: $\Delta h(\Delta x)$ on a log-log plot exhibits a power law behaviour with exponent $\beta \simeq 0.55$ from $\Delta x \sim 0.06 \mu \mathrm{m}$ to $\Delta x \sim 800 \mu \mathrm{m}$, i.e. over more than four decades of length scales. Inset: $\Delta h(\Delta z)$ on a log-log plot exhibits a power law behaviour with exponent $\zeta \simeq 0.54$ from $\Delta z \sim 0.3 \mu \mathrm{m}$ to $\Delta x \sim 300 \mu \mathrm{m}$.

$\Delta h(\Delta z))$ exhibits a power law increase with a roughness exponent $\beta \approx 0.55$ (respectively, $\zeta \approx 0.54$ ).

In a second step, we compute the statistical distribution of height increments $\Delta h$ between points separated by a distance $\Delta z$ along the direction $z$ of the crack front. The resulting probability distribution function $P(\Delta h)$ is plotted in fig. 3a. If our profiles were perfectly mono-affine, i.e. if all the moments of the heights distribution were characterized by the same roughness exponent $\zeta \approx 0.55$, the statistics of $h$ increments at scale $\Delta z$ would involve a unique relevant length $\Delta z^{\zeta}$. In this case, the rescaling $\Delta h \rightarrow \Delta h / \Delta z^{\zeta}$ and $P \rightarrow \Delta z^{\zeta} P$ would ensure a collapse of all the curves onto a single master curve. Such a collapse is clearly not observed in the present case (fig. 3b). Hence, $\Delta z^{\zeta}$ is not the only relevant length at scale $\Delta z$, which reveals a clear multi-affine behaviour. Fracture surfaces in metallic glass exhibit multi-affine scaling contrary to what is commonly observed in other materials [26-28]. However, we suspect that the tails of the distribution are mostly due to high cliffs which are the signature of overhangs that our technique cannot capture (see discussion below).

Cavity formation. - We use the Frasta method $[29,30]$ to reconstruct cavity formation during the fracture process. This method is based on the observation that for ductile materials, the mismatch between the opposite fracture surfaces (see fig. 1 and fig. $4 \mathrm{~b}$ ) is due to the formation of damage cavities ahead of the crack front. These cavities leave their imprints on the fracture surfaces which then do not perfectly fit one another.

For each pair of opposite fracture surfaces (magnifications $\times 100, \times 300$ and $\times 3000$ ), we first cross-correlate the two surfaces in order to get the best possible matching. Prior to the computation of the cross-correlation, an 

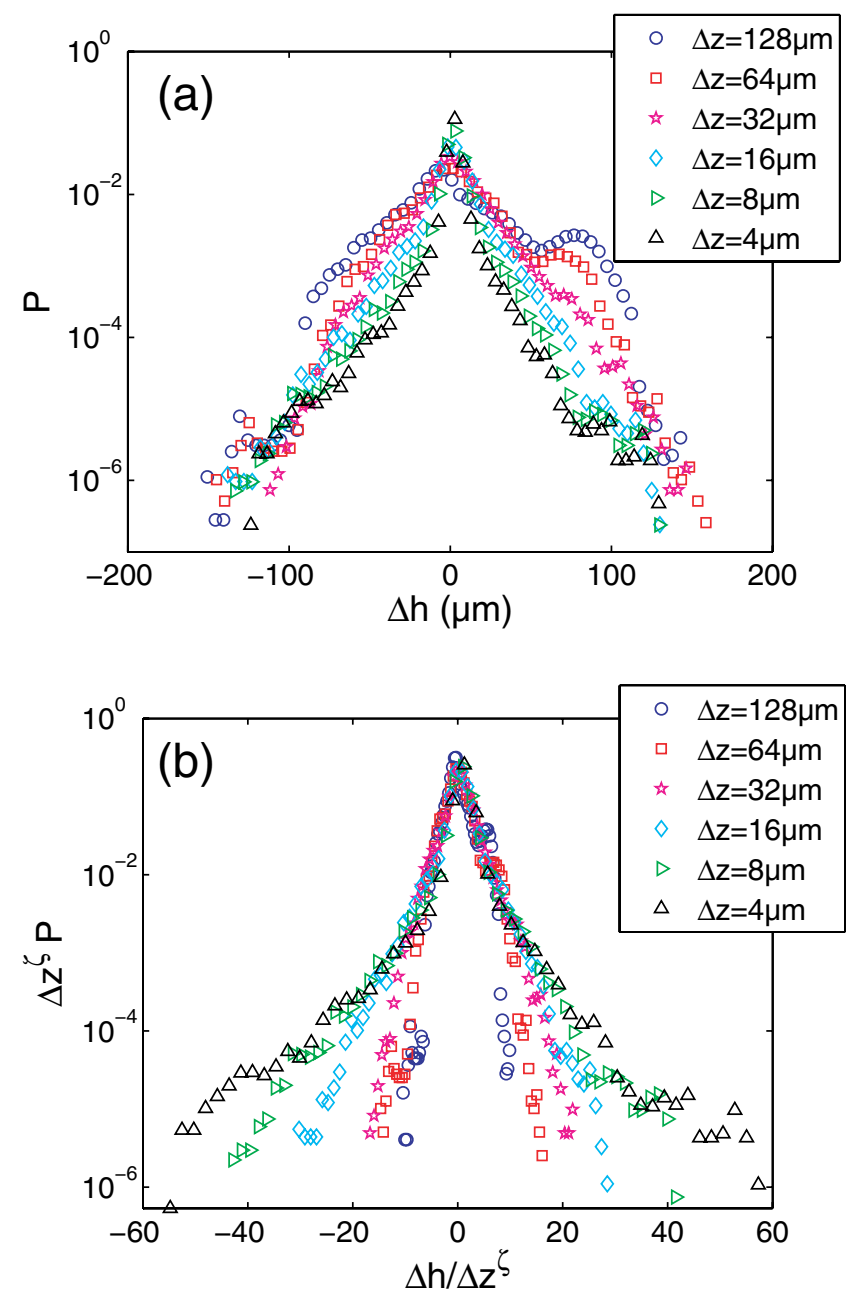

Fig. 3: Top: probability distribution function $P$ of height increments $\Delta h$ for various values of $\Delta z$. Bottom: $P \Delta z^{\zeta}$ as a function of $\Delta h / \Delta z^{\zeta}$. There is no collapse of the curves.

average plane is substracted from each surface. Then, for each magnification, the positions of the upper and lower fracture surfaces are inverted, i.e. the upper surface is virtually placed underneath the lower one, and then "pulled" upwards so that the two surfaces start interpenetrating each other (see fig. 4). For each distance $\Delta y$ between the two surfaces $(\Delta y=0$ corresponding to the same average plane for the two surfaces), we substract one surface from the other, a cavity being defined as the zone where the upper fracture surface is higher than the lower one (see fig. 4).

For magnification $\times 3000$, the total number of cavities per unit area is plotted as a funtion of distance $\Delta y$ between the two opposite surfaces in fig. 5. It shows a maximum for a distance $\Delta y \simeq-0.7$ to $-0.4 \mu \mathrm{m}$ which corresponds to a cavity surface fraction of $\sim 0.24$. Figure 6 shows that: i) the curve in fig. 5 is only weakly asymmetric with respect to the maximum, since both curves shown here are plotted as functions of the absolute value of $Y=\Delta y+0.6(\mu \mathrm{m})$; ii) the surface fraction of cavities behaves as a power law
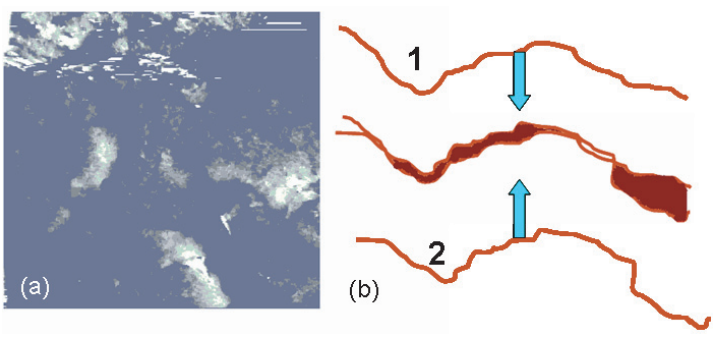

Fig. 4: (a) Cavities in grey levels for $\Delta y=-0.6 \mu \mathrm{m}$. (b) Sketch of the method: surface 1 is the lower fracture surface and surface 2 is the upper one. Their relative positions are first inverted (surface 1 is put on top of surface 2 as shown here), and they are subsequently pulled toward each other. Whenever surface 1 comes under surface 2 , this is interpreted as a damage cavity (dark filling in the sketch above).



Fig. 5: Evolution of the number of cavities per unit area as a function of distance $\Delta y$ between the two fracture surfaces. The maximum corresponds to a coverage of $23.85 \%$.



Fig. 6: Log-log plot showing the power law decrease of the number of cavities per unit area as a function of $|\Delta y+0.6|$. Inset: exponential decrease of the same quantity $\propto \exp ^{-|\Delta y+0.6| / 0.75}$ for smaller values of $\Delta y$.

with exponent of -1.6 for distances such that $|Y|$ is larger than $\sim 1 \mu \mathrm{m}$, while iii) (see inset) the surface fraction decreases as $\exp ^{-\left|\frac{Y}{0.75}\right|}$ closer to the maximum. 


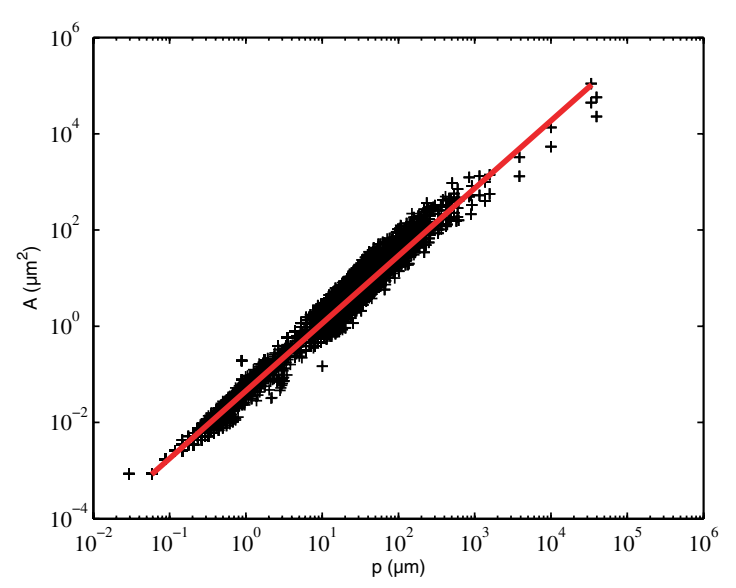

Fig. 7: Cavity area vs. cavity perimeter. The best fit is a power law with exponent $2 / d_{F} \approx 1.40$; Hence $d_{F} \approx 1.43$ is the fractal dimension of the islands.

The morphology of the cavities is explored by studying both their areas and their perimeters. The area and the perimeter of each island corresponding to the intersection of a given cavity with the $x z$-plane is recorded for several values of $\Delta y$ and for the three sets of opposite fracture surfaces. Figure 7 shows a plot of areas vs. perimeters for all these islands.

Finally, we compute the cumulative distribution functions of areas and perimeters, $P(A)$ and $P(p)$, respectively. Both are obtained by ranking the areas and the perimeters, respectively, in ascending orders. $P(A)$ (respectively, $P(p)$ ) is the rank plotted as a function of $A$ (respectively, $p)$. Figure 8 and its inset show $P(A)$ and $P(p)$ respectively, for magnification $\times 3000$, and a distance $\Delta y=$ $-0.6 \mu \mathrm{m}$ between the opposite surfaces. Both $P(A)$ and $P(p)$ decrease as power laws with exponents $-\alpha_{A}=-0.62$ and $-\alpha_{p}=-0.88$.

Discussion. - We show in this paper that fracture of the Vitreloy 1 metallic glass occurs mainly through cavity formation and coalescence: Damage cavities are indeed printed on the fracture surfaces, which, as a consequence, do not match each other. We show that the number of these cavities reaches a maximum for a given distance $|\Delta y|=0.6 \mu \mathrm{m}$ between the two opposite fracture surfaces. This corresponds to the critical point where damage cavities start coalescing with each other, for a surface fraction $\approx 23.9 \%$ of cavities within the $x z$-plane. This critical point is characterized by a power law variation of the number of cavities.

Damage cavities have a fractal surface. It is characterized by plotting the areas of their intersections with the $x z$-plane as a function of their perimeters. Because only the surface of damage cavities is fractal, these intersections have a fractal contour, but they are dense objects. Hence, if $l$ is a linear dimension of such an island, its area scales as $A \propto l^{2}$, while its perimeter scales as $p \propto l^{d_{F}}$, where $d_{F}$ is the fractal dimension of the island. As a consequence, the relationship between area and perimeter is



Fig. 8: Cumulative distribution function $P(A)$. The best fit is a power law with exponent $-\alpha_{A} \approx-0.62$. Inset: cumulative distribution functions $P(p)$. The best fit is a power law with exponent $-\alpha_{p} \approx-0.88$.

non-trivial: $A \propto p^{2 / d_{F}}$. This is how we interpret the power law increase shown in fig. 6 , which gives: $d_{F}=1.43$. This value is compatible with both roughness indices measured on the fracture surfaces (see fig. 2 ), since: $\zeta \approx \beta \approx 0.57=$ $2-d_{F}$. This means that the fractal nature of damage cavities is what controls the roughness of the fracture surface, which can be viewed as composed of the adjacent imprints of such cavities.

The obtained value of $d_{F}$ is compatible with exponents $\alpha_{A}$ and $\alpha_{p}$. As a matter of fact, the following relation holds:

$$
\frac{\mathrm{d} P(A)}{\mathrm{d} A} \mathrm{~d} A=\frac{\mathrm{d} P(p)}{\mathrm{d} p} \mathrm{~d} p
$$

or: $2 \alpha_{A}=\alpha_{p} d_{F}$. We find: $2 \alpha_{A} \approx 1.24$ and: $d_{F} \alpha_{p} \approx 1.26$.

Although our fracture surfaces are anisotropic to the eye (see fig. 1), they are actually isotropic from the point of view of roughness exponents. However, fig. 2 shows that the bounds of the scaling domains are quite different for the $x$ and $z$ directions: $\Delta h(\Delta x)$ scales as $\Delta x^{\beta}$ for $0.06 \mu \mathrm{m}<\Delta x<800 \mu \mathrm{m}$, while $\Delta h(\Delta z)$ scales as $\Delta z^{\zeta}$ for $0.3 \mu \mathrm{m}<\Delta z<300 \mu \mathrm{m}$.

Although these power laws extend over a large range of length scales, the fracture surfaces do not exhibit a monoaffine, but rather a clear multi-affine behaviour. The fact that they are constituted by the coalescence of cavities implies that overhangs could be present. These overhangs cannot be captured with the present experimental method, nor with methods such as profilometry, or, at smaller length scales, Atomic Force Microscopy. With all these methods, overhangs are seen as high "cliffs", the presence of which may be responsible for the observed multi-affinity. New observations involving a cut of the samples within planes parallel to $x y$ or $y z$ should be performed in order to access the statistics of these overhangs.

On the other hand, the anisotropy of the damage cavities themselves still has to be studied. As a matter of fact, it was observed in [22] that in aluminium alloys, while "small" damage cavities were isotropic with a roughness index close to 0.5 , "larger" cavities were anisotropic, with 
a roughness index $\zeta \simeq 0.8$ in the direction perpendicular to crack propagation. In ductile materials, small damage cavities enlarge through surface growth, while large cavities, which are closer to penny-shaped cracks enlarge because their front propagates. The difference in growth mechanisms might be at the origin of the difference in morphology. In the present case, surface growth of cavities might be responsible for the observed isotropic roughness with exponent $\simeq 0.55$, in contrast with what is observed for the propagation of a crack front which generates an anisotropic fracture surface with exponents $\beta \simeq 0.6$ and $\zeta \simeq 0.75$.

It has also been suggested [31] that the anisotropy of fracture surfaces was not intrinsic, but due to the introduction of a precrack in the specimens. Our fracture surfaces are actually isotropic despite the presence of an initial notch. Of course, since our images were taken far from the edges of the specimen, we may have missed the transient behaviour where, very close to the notch, the surface has to be anisotropic.

Further experiments with longer and sharper notches will be performed on Vitreloy 1 to check whether the isotropy is a property of our material, or if it is linked to the isotropy of loading, with no imposed initial stress concentration. Conversely, experiments on cylindrical specimens of steel will be made, since it has been shown that on this material, fracture surfaces obtained from precracked Compact Tension specimens are anisotropic [32].

It was also suggested that in metallic glasses, the rise of temperature at the crack tip is such that the material melts locally [33]. This should modify the morphology of the observed cavities. A better understanding of a possible melting should arise from a comparison with metallic fracture surfaces.

As a matter of fact, a roughness index close to the one reported here $(i . e . \approx 0.55)$ was observed on metallic fracture surfaces [8-10] at small length scales/low crack velocities. It was shown that the crossover length separating this regime from the $\zeta \simeq 0.75$ regime at higher length scales was inversely proportional to the average crack velocity $V$. If one assumes that the extension velocity of the damage cavity closer to the main crack is independent of $V$, it is easily shown that the cavity size at coalescence scales as $1 / V$. In this picture, for low enough values of $V$, one might be able to observe the $\zeta^{\prime} \approx 0.55$ isotropic regime at very small length scales, the anisotropic " $0.75 / 0.6$ " regime within the process zone, and the (slightly) anisotropic "0.4/0.5" regime at larger length scales [32], where the material can be well described by linear elasticity [12].

$$
* * *
$$

EB wishes to thank J.-P. Bouchaud for inspiring discussions, and for his constant support. She is also indebted to Y. CHAMPION for an interesting discussion, and for pointing out ref. [33].

\section{REFERENCES}

[1] Mandelbrot B. B., Passoja D. E. and Paullay A. J., Nature, 308 (1984) 721.

[2] Bouchaud E., J. Phys.: Condens. Matter, 9 (1997) 4319.

[3] Bouchaud E., Surf. Rev. Lett., 10 (2003) 797.

[4] Alava M. J., Nukala P. K. and Zapperi S., Adv. Phys., 55 (2006) 349.

[5] Bouchaud E., Lapasset G. and Planés J., Europhys. Lett., 13 (1990) 73.

[6] Dauskardt R. H., Haubensk F. and Ritchie R. O., Acta Metall. Mater., 38 (1990) 143.

[7] Morel S., Lubet T., Pouchou J.-L. and Olive J.-M., Phys. Rev. Lett., 93 (2004) 065504.

[8] Daguier P. et al., Phys. Rev. E, 53 (1996) 5637.

[9] Daguier P. et al., Phys. Rev. Lett., 78 (1997) 1062.

[10] Bouchaud E. and Naveos S., J. Phys. I, 5 (1995) 547.

[11] Ponson L., Bonamy D. and Bouchaud E., Phys. Rev. Lett., 96 (2006) 035506.

[12] Bonamy D., Ponson L., Prades S., Bouchaud E. and Guillot C., Phys. Rev. Lett., 97 (2006) 135504.

[13] Ponson L., Bonamy D. and Barbier L., Phys. Rev. B, 74 (2006) 184205.

[14] Schmittbuhl J., Schmitt F. and Scholz C., Geophys. Res., 100 (1995) 5953.

[15] Mourot G., Morel S., Bouchaud E. and Valentin G., Phys. Rev. E, 71 (2005) 016136; Int. J. Fract., 140 (2006) 39.

[16] Weiss J., Eng. Fract. Mech., 68 (2001) 1975.

[17] Engøy T., Måløy K. J., Hansen A. and Roux S., Phys. Rev. Lett., 73 (1994) 834.

[18] Morel S., Schmittbuhl J., López J. M. and Valentin G., Phys. Rev. E, 58 (1998) 6999; Morel S., Mourot G. and Schmittbuhl J., Int. J. Fract., 121 (2003) 23.

[19] MÅLøY K. J. et al., Phys. Rev. Lett., 68 (1992) 213.

[20] Ponson L., Auradou H., Vie P. and Hulin J.-P., Phys. Rev. Lett., 97 (2006) 125501.

[21] Morel S., Bonamy D., Ponson L. and Bouchaud E., Phys. Rev. E, 78 (2008) 016112.

[22] Paun F. and Bouchaud E., Int. J. Fract., 121 (2003) 43.

[23] Peker A. and Johnson W. L., Appl. Phys. Lett., 63 (1993) 2342.

[24] Lu J., Ravichandran G. and Johnson W. L., Acta Mater., 51 (2003) 3429.

[25] Pouchou J.-L. et al., Mikrochim. Acta, 139 (2002) 135.

[26] Bouchaud E., Lapasset G., Planés J. and Naveos S., Phys. Rev. B, 48 (1993) 2917.

[27] Santucci S. et al., Phys. Rev. E, 75 (2006) 016104.

[28] Ponson L. et al., Phys. Rev. B, 76 (2007) 036108.

[29] Kobayashi T. and Shockey D. A., Metall. Trans. A, 18 (1987) 1941.

[30] Мiyamoto H., Kikuchi M. and Kawazoe T., Int. J. Fract., 42 (1990) 389.

[31] Bouchbinder E., Procaccia I. and Sela S., Phys. Rev. Lett., 95 (2005) 255503-1.

[32] Guerra-Amaro C. et al., in preparation (2008).

[33] Lewandowski J. J. and Greer A. L., Nat. Mater., 5 (2006) 15. 